\title{
Clinical Significance of Hyperdense Area after Endovascular Therapy in Patients with Acute Ischemic Stroke: A Systematic Review and Meta-Analysis
}

\author{
Qianmei Jiang Jie Hou Jian Ge Zhichao Huang Huaishun Wang \\ Zhiliang Guo Yongjun Cao Shoujiang You Guodong Xiao
}

Department of Neurology and Suzhou Clinical Research Center of Neurological Disease, The Second Affiliated

Hospital of Soochow University, Suzhou, China

\section{Keywords}

Hyperdense area · Acute ischemic stroke · Endovascular

therapy · Meta-analysis

\begin{abstract}
Objective: We performed a systematic review and metaanalysis to investigate the clinical significance of hyperdense area after thrombectomy in patients with acute ischemic stroke (AIS). Methods: We searched Ovid MEDLINE(R) and Epub Ahead of Print, In-Process and other Non-Indexed, Cochrane Library Clinical Controlled Trials and Embase from inception to September 2020 and collected the cohort and case-control studies about the clinical significance of hyperdense area on different types of computed tomography (CT) after thrombectomy in patients with AIS. Outcomes were poor functional outcome (modified Rankin Scale [mRS] Score 3-6 at discharge or 90-day), mortality and subtypes of hemorrhage according to the European Cooperative Acute Stroke Study (ECASS). Results: 1,999 patients from 16 studies were included in this meta-analysis. Pooled results indicated higher risk of symptomatic intracerebral hemorrhage (odds ratio $[\mathrm{OR}]=3.02 ; 95 \%$ confidence interval $[\mathrm{Cl}]$ 1.84-4.95; $p<$ $0.0001, R^{2}=0 \%$ ) in patients with hyperdense area, and the
\end{abstract}

karger@karger.com

(C) 2021 S. Karger AG, Basel

www.karger.com/ced

Karger ${ }^{\prime}=$ subtype of parenchymal hematoma as well. There was also higher odds of poor functional outcome based on the mRS $3-6$ at discharge or 90 -day $(\mathrm{OR}=1.92 ; 95 \% \mathrm{Cl} 1.35-2.73 ; p=$ $\left.0.0003, L^{2}=31 \%\right)$ and mortality $(\mathrm{OR}=2.06 ; 95 \% \mathrm{Cl} 1.41-3.02$; $p=0.0002, I^{2}=0 \%$ ) in patients with hyperdense area after thrombectomy compared with those without hyperdense area. Conclusions: Our results indicated that the presence of hyperdense area on CT after thrombectomy was associated with high risk of symptomatic intracerebral hemorrhage, poor functional outcome, as well as mortality in patients with AIS. However, further studies were needed to confirm these results. The meta-analysis was conducted in adherence with the PRISMA Statement and was registered at the International Prospective Register of Systematic Reviews (CRD42020164165). To the best of our knowledge, this study is the first meta-analysis investigating the effect of hyperdense area after endovascular therapy in patients with AIS.

(c) 2021 S. Karger AG, Basel

Qianmei Jiang and Jie Hou contributed equally to this work. Shoujiang You and Guodong Xiao contributed equally as corresponding authors. 


\section{Introduction}

Thrombectomy has evolved substantially in recent years and has become the cornerstone of treatment strategy for patients with proximal anterior circulation occlusion due to more advanced neuroimaging patient selection [1-4]. A meta-analysis from 5 randomized trials has suggested that modern endovascular thrombectomy added to best medical therapy more than doubles the odds of good functional outcome compared with best medical therapy alone [5].

Hyperdense area is common on computed tomography (CT) of acute ischemic stroke (AIS) patients after thrombectomy, which may be a sign of contrast medium leakage simply, bleeding, or a combination of both [6-8]. Hyperdense area exists frequently on CT after thrombectomy immediately, but typically, it is often weakened or disappeared after a few hours or days, thus being likely to be misdiagnosed as bleeding [9]. Furthermore, compared to patients without hyperdense area, patients with hyperdense area have longer onset to reperfusion time, and more numbers of thrombectomy devices pass as breaking the blood-brain barrier [10-12]. It is helpful to determine the earlier antiplatelet drug use for preventing vascular reocclusion caused by endothelium injury if the hyperdense area can be well distinguished from hemorrhage [13-15]. Since then, the ability of hyperdense area on CT after thrombectomy for predicting hemorrhage was not consistent in published studies. Several studies reported that the presence of hyperdense area may be associated with higher risks of hemorrhage [16-20]. Some studies have indicated that only specific types of hyperdense area were related to specific types of intracranial hemorrhage $[12,21-28]$. While, no significant association was found between hyperdense area and hemorrhage in other studies $[29,30]$. In addition, it has been unknown whether hyperdense area after endovascular therapy was associated with poor functional outcome. The current mainstream view was that the presence of hyperdense area might be predictive of unfavorable functional outcome [10-12] in AIS patients, whereas some indicated it had no effect on functional outcome [16, 18]. Contrarily, Wildenhain et al. [31] supported rapid clearing of these lesions may be a positive prognostic sign. Hence, uncertainties still remain in the predictive value of hyperdense area visualized on the postinterventional CT scan for detection of hemorrhage and poor functional outcome in AIS patients.

As a consequence, we aimed to perform this metaanalysis to investigate the association between hyperdense area after endovascular therapy and hemorrhage, poor functional outcome as well as mortality in patients with AIS. We hope it can give an implication for optimization of antithrombotic therapy strategy for patients with hyperdense area after endovascular treatment.

\section{Methods}

\section{Databases and Sources}

We identified all published studies about clinical significance of hyperdense area after thrombectomy in patients with AIS using Ovid MEDLINE(R) and Epub Ahead of Print, In-Process and other Non-Indexed, Cochrane Library Clinical Controlled Trials and Embase from the establishment of the database to September 2020 without any language restriction. The search terms were "cerebral ischemia" OR "stroke" OR "brain infarction" OR "cerebrovascular disease" AND "thrombectomy" OR "endovascular" OR "mechanical thrombolysis" AND "computed tomography" or "CT" AND "contrast" OR "parenchymal hyper-density" OR "hyper-attenuated" OR "iodine." We also manually searched the reference lists of the relevant articles to identify additional studies that were not included in the initial literature searches.

\section{Study Selection}

Two investigators (Q.M.J. and J.H.) independently reviewed each entry. Disagreements were resolved by consensus or with the help of the senior investigator (G.D.X.). Matched cohort studies and case-control studies were included. Case reports and reviews were excluded. The identified studies were then evaluated with the following inclusion criteria: (1) AIS patients who underwent thrombectomy therapy and received CT after treatment; (2) the study reported the contrast staining or hyperdense area on CT after treatment and compared the outcomes of the patients with hyperdense area and those without hyperdense area; (3) recorded baseline characteristics information, including study locations, mean age, proportion of male, National Institutes of Health Stroke Scale (NIHSS), definition of hyperdense area and so on; (4) Sufficient data of modified Rankin Scale (mRS) score at 3 months or discharge, symptomatic intracranial hemorrhage $(\mathrm{sICH})$ or other subtypes of hemorrhage according to European Cooperative Acute Stroke Study (ECASS) [32] criteria after CT examination.

\section{Data Extraction}

Two investigators (Q.M.J. and J.G.) independently extracted all baseline data and main outcomes from each eligible study: (1) general data: name of author, year of publication, number of cases, location of lesion, mean age, proportion of male, NIHSS, definition of hyperdense area, and available outcome index. (2) main outcomes: numbers of patients with sICH, parenchymal hematoma type 2 ( $\mathrm{PH} 2)$, parenchymal hematoma $(\mathrm{PH})$, and hemorrhage transformation (HT) according to the criteria of ECASS [32]; numbers of patients with poor functional outcome, which was defined as mRS score 3-6 at discharge or 90-day; proportion of mortality.

\section{Quality Assessment}

To evaluate the risk of bias, 2 investigators (Q.M.J. and S.J.Y.) independently assessed each article according to Newcastle-Ottawa Quality Assessment Scale for nonrandomized studies [33]. 
Fig. 1. Flow diagram of literature screening and selection process.

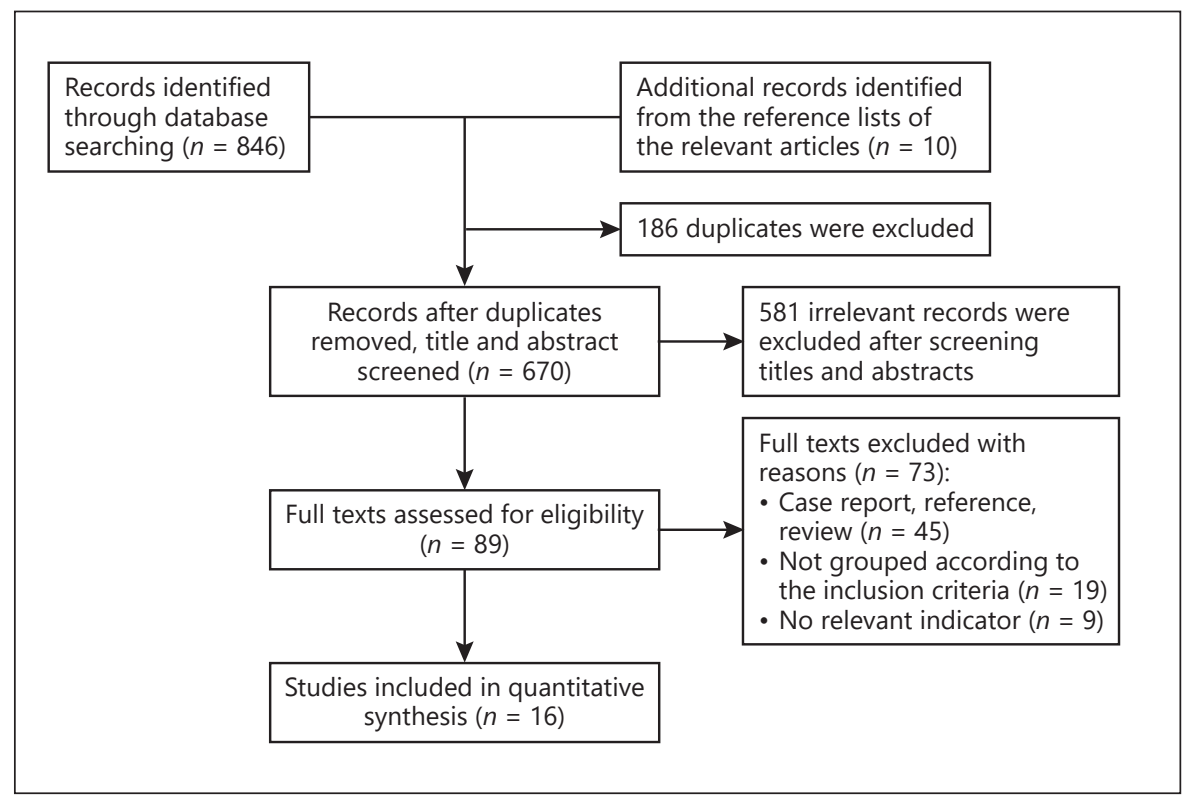

Case-control studies were assessed basing on 3 aspects: selection of cases, comparability of cases, and ascertainment of exposure. Cohort studies were assessed based on patient selection, study comparability, and outcome. Disagreements were resolved by consensus or with the help of the senior investigator (GDX).

\section{Data Synthesis and Analysis}

Meta-analysis was carried out by RevMan 5.3 provided by Cochrane Collaboration network. The heterogeneity included in the studies was tested by $I^{2}$ test. If $p<0.1$ and $I^{2}>50 \%$, random-effect model was used. If not, the fixed-effect model was employed for combined analysis. Subgroup analysis was used to detect the sources of heterogeneity based on location of lesion (anterior or posterior stroke), definition of hyperdense area, and the types of CT examination. We applied odds ratio (OR) to represent the enumeration data. Measurement data would be represented by mean difference and $95 \%$ confidence interval (95\% CI). Sensitivity analysis was conducted by sequential exclusion of one study at a time to assess for a significant change in the summary OR. When more than 10 studies are included, publication bias would be evaluated by the asymmetry of the funnel plot.

\section{Results}

\section{Characteristics of Included Studies}

As shown in the flow diagram (Fig. 1), we identified 846 articles at first. Finally, 16 studies comprising 1,999 patients were included for quantitative synthesis. The study characteristics are shown in Table 1, and the associated definitions used in included studies were displayed in the see online suppl. materials; for all online suppl. material, see www.karger.com/doi/10.1159/000515410 (on- line Suppl. Table 1). Eleven case-control studies and 5 cohort studies were included with the sample size ranging from 62 to 226 . The selected studies were conducted between 2001 and 2020 in 6 countries. Of the 16 studies included, 8 studies $[10,11,16,17,19,21-23]$ reported the relationship between post-interventional hyperdense area and poor functional outcome, while 15 studies outlined the correlation between post-interventional hyperdense area and HT, along with 9 studies $[10,11,16,19$, $20,23,24,29,34]$ for sICH, 8 studies $[10,11,18,22,26$, $27,29,34]$ for $\mathrm{PH}$ and 6 studies [17, 18, 22, 27, 29, 34] for $\mathrm{PH} 2$. Hemorrhagic transformation types were defined as the criteria used in the ECASS [32] test. Among 9 studies validating relationship between post-interventional hyperdense area and sICH, 6 studies defined sICH as any ICH with an increase of 4 points or more in NIHSS. The Newcastle-Ottawa Scale (NOS) was used to assess study quality. In this meta-analysis, 4 studies attained 7 points on the NOS scale and the remaining 12 studies attained 8 points.

\section{Relationship between Post-Interventional Hyperdense} Area and Hemorrhage

Hyperdense area significantly increased the risk of sICH (OR = 3.02; 95\% CI 1.84-4.95; $p<0.0001, I^{2}=0 \%$, Fig. 2a $)$ and $\mathrm{PH}(\mathrm{OR}=6.54 ; 95 \% \mathrm{CI} 1.68-25.46 ; p=0.007$, $I^{2}=85 \%$, Fig. $2 \mathrm{~b}$ ), but no association was found between hyperdense area and $\mathrm{PH} 2(\mathrm{OR}=4.55 ; 95 \% \mathrm{CI} 0.85-24.39$; $p=0.08, I^{2}=66 \%$, Fig. 2c). Moreover, significant associations between hyperdense area and sICH were also ob- 


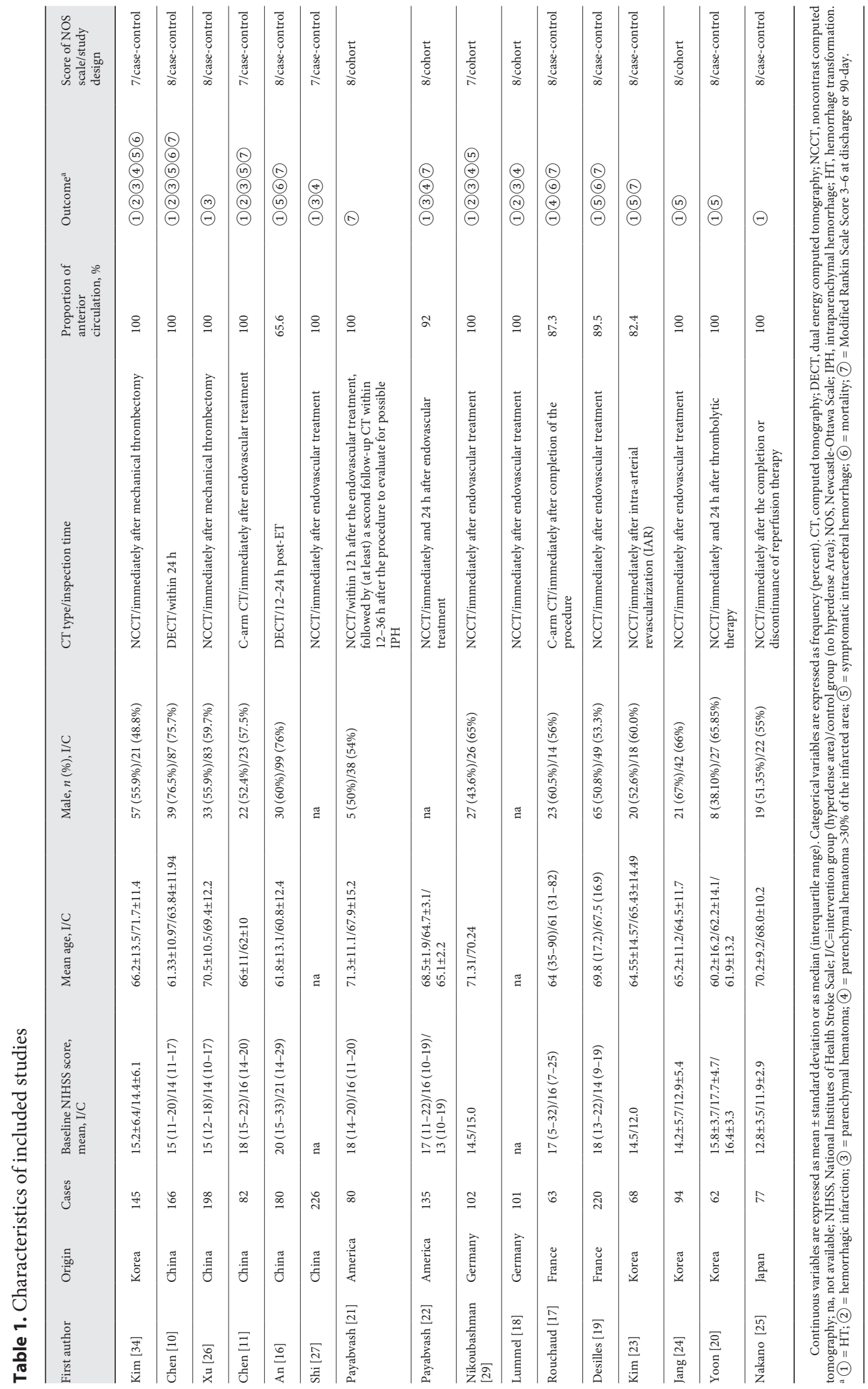




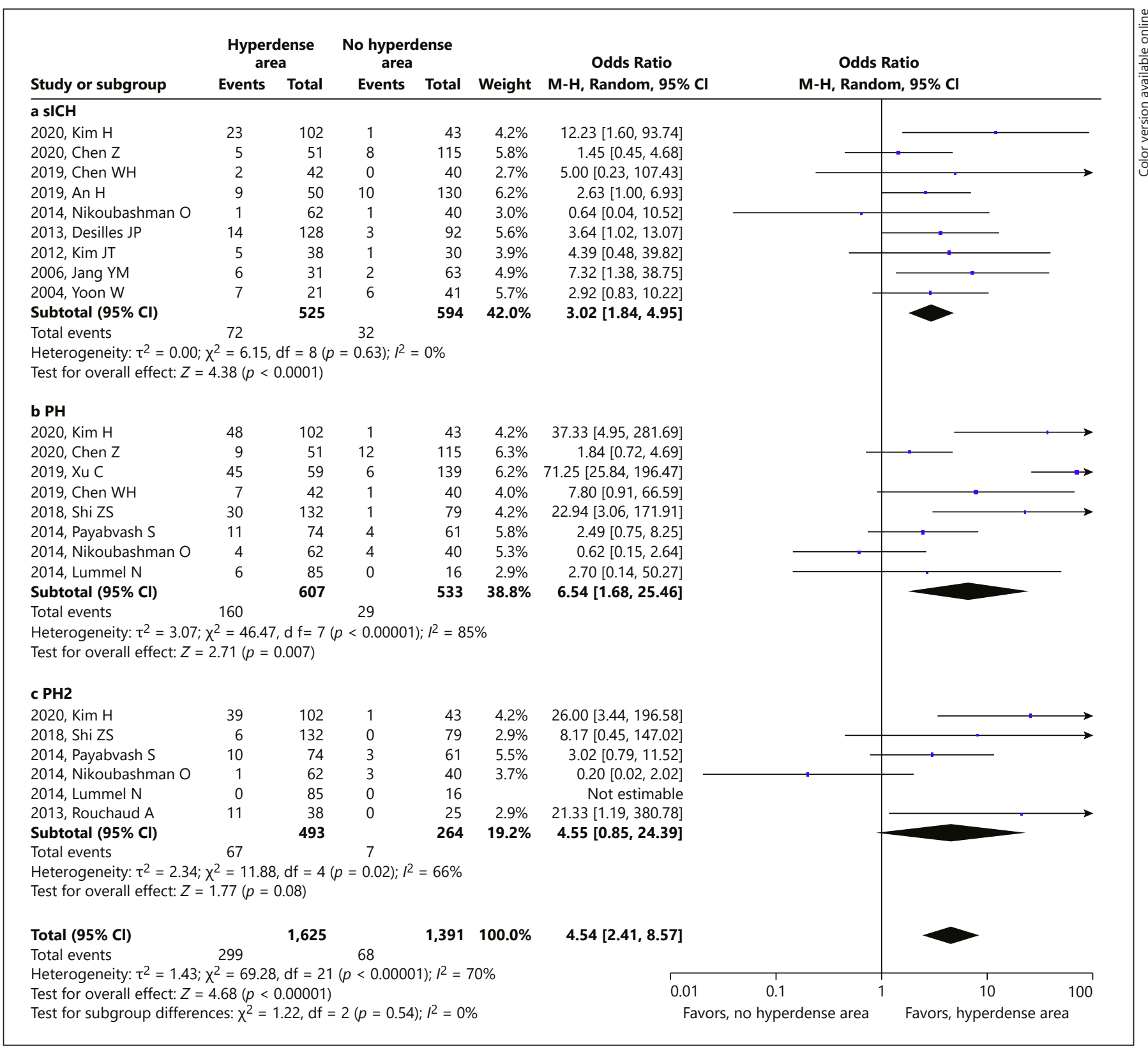

Fig. 2. Forest plot for $\mathrm{sICH}, \mathrm{PH}, \mathrm{PH} 2$ in patients with hyperdense area after thrombectomy compared with those without hyperdense area. $\mathrm{PH}$, parenchymal hematoma; $\mathrm{PH} 2$, parenchymal hematoma type 2.

served in subgroups based on different locations (online suppl. Fig. 1a, b) and specific Hounsfield unit of hyperdense area (online suppl. Fig. 1c). Besides, there were more HT $(\mathrm{OR}=5.99 ; 95 \%$ CI 3.29-10.93; $p<0.00001$, $I^{2}=80 \%$, online suppl. Fig. 2) events in the hyperdense area group than no hyperdense area group. And the association between hyperdense area group and HT was still significant in the subgroup analysis, including differ- ent types of CT (online suppl. Fig. 2b, d), the time from CT scan after thrombectomy (online suppl. Fig. 2e), the persisted time of hyperdense area (online suppl. Fig. 2f) as well as the maximum CT unit (online suppl. Fig. 2g). The symmetrical funnel plot of the 15 studies (online suppl. Fig. 3) that reported the outcome of HT did not show publication bias. 


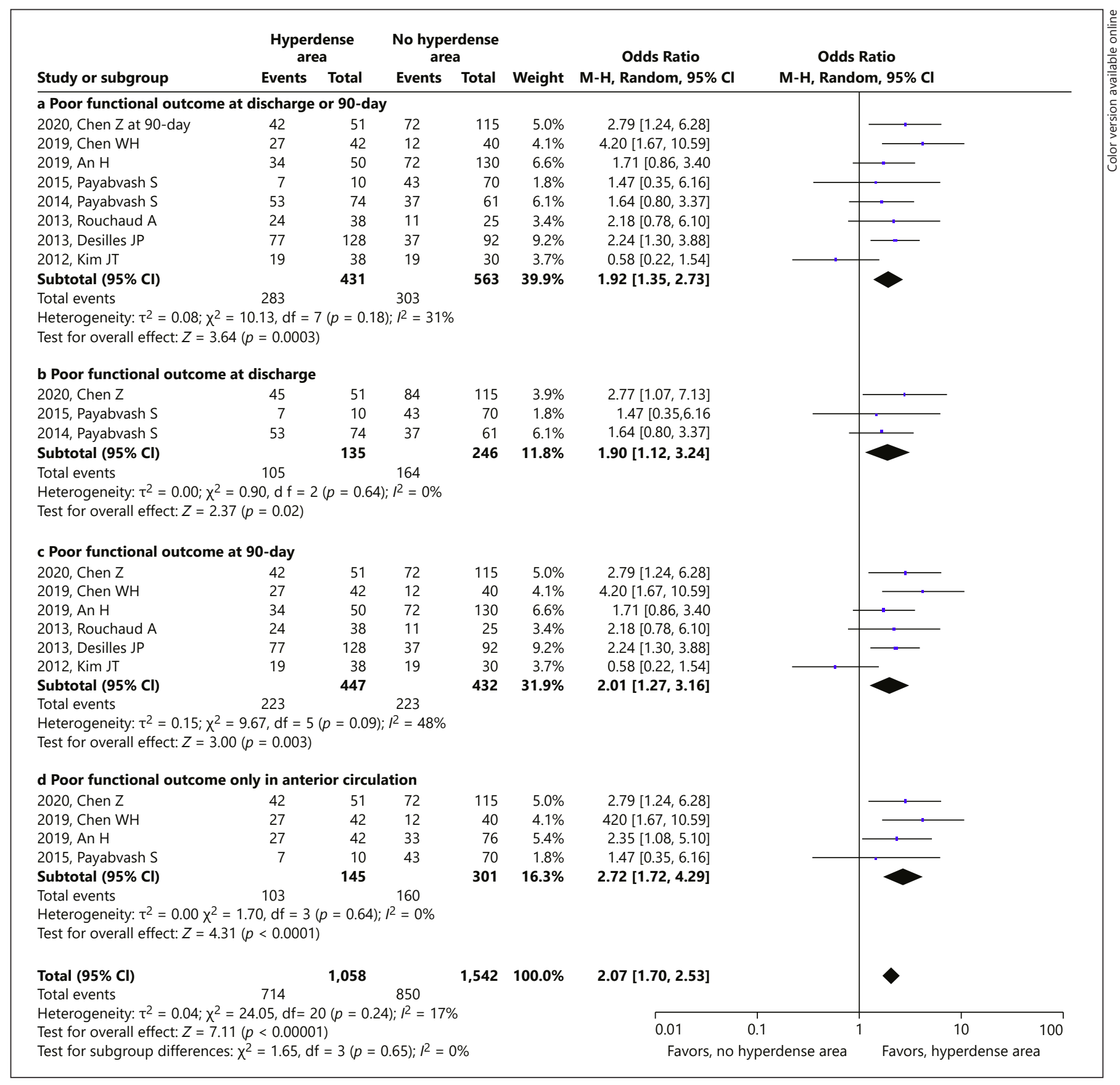

Fig. 3. Forest plot for poor functional outcome in patients with hyperdense area after thrombectomy compared with those without hyperdense area.

\section{Relationship between Post-Interventional Hyperdense Area and Poor Functional Outcome}

A meta-analysis of 8 eligible studies including 994 patients illustrated that patients with hyperdense area had a higher rate of poor functional outcome at discharge or 90 -day than those without hyperdense area $(\mathrm{OR}=1.92$;
95\% CI 1.35-2.73; $p=0.0003, I^{2}=31 \%$, Fig. 3a). The subgroup analyses included studies with only poor functional outcome at discharge $(\mathrm{OR}=1.90 ; 95 \%$ CI 1.12-3.24; $p=0.02, I^{2}=0 \%$, Fig. $3 \mathrm{~b}$ ), only poor functional outcome at 90-day $\left(\mathrm{OR}=2.01 ; 95 \%\right.$ CI $1.27-3.16 ; p=0.003, I^{2}=$ $48 \%$, Fig. 3c) and only in anterior circulation (Fig. 3d). 


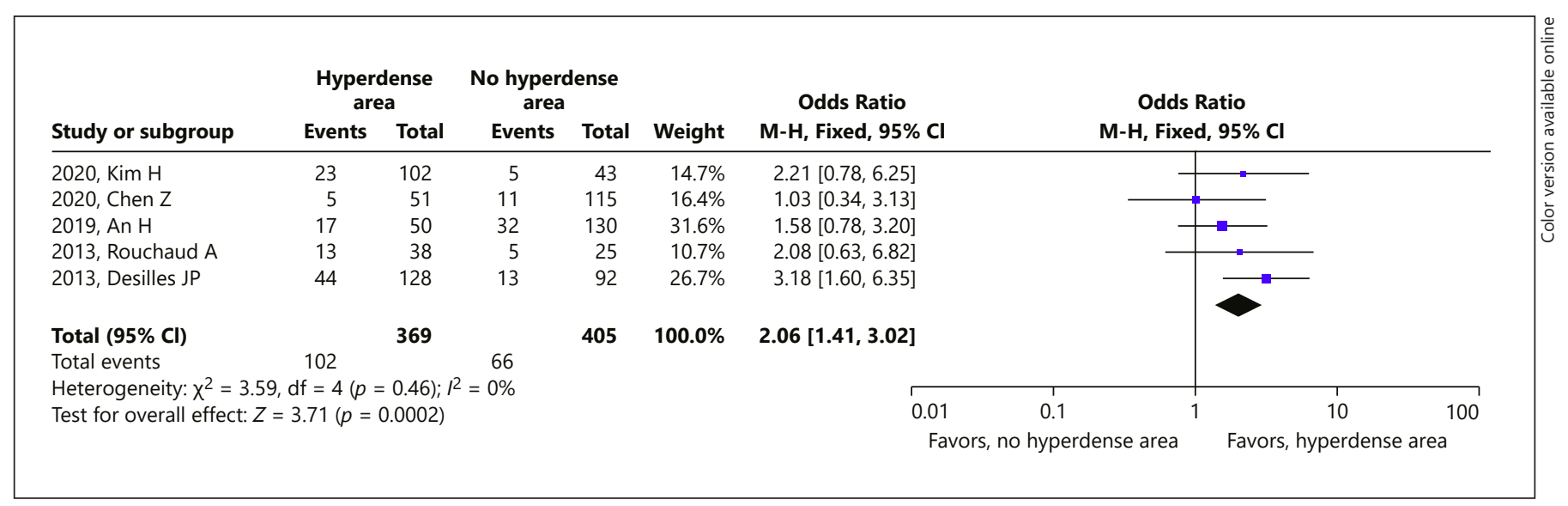

Fig. 4. Forest plot for mortality in patients with hyperdense area after thrombectomy compared with those without hyperdense area.

Owing to both contrast agent extravasation and hemorrhage displaying high-density lesions on mixed energy images of dura-energy CT, we regarded them as one group in our meta-analysis when it comes to its prognostic value. In sensitivity analyses, results remain consistent with the full analysis when sequential omitting one study.

\section{Relationship between Post-Interventional Hyperdense}

Area and 3- to 12-Month Mortality

A meta-analysis of 5 eligible studies including 774 patients illustrated that patients with hyperdense area had a higher rate of 3- to 12-month mortality than those without hyperdense area $(\mathrm{OR}=2.06 ; 95 \%$ CI $1.41-3.02 ; p=$ $0.0002, I^{2}=0 \%$, Fig. 4 ). In sensitivity analyses, sequential omitting one study at a time from the meta-analysis showed results consistent with ordinary outcome.

\section{Discussion}

This current meta-analysis consisted of 16 included studies (1,999 patients), indicating that hyperdense area on CT after thrombectomy was significantly associated with high risk of symptomatic intracerebral hemorrhage and poor functional outcome in patients with AIS. Moreover, higher odd risk of mortality was found in patients with hyperdense area on CT compared to patients without hyperdense area.

The term "hyperdense area," known as "intraparenchymal hyper-attenuation (IPH)" or "postinterventional cerebral hyper-density (PCHD)," could also be divided into "contrast enhancement" and "contrast extravasation" due to the different duration of hyperdense area and their clinical significance [20]. As early as 1993, Komiyama et al. [35] have reported that contrast media extravasation in the basal ganglia region occurred in 2 patients with local intracarotid fibrinolysis. In recent years, in order to judge the changes of patients' condition and optimize treatment plans, the reports about this kind of hyperdense area have also been increasing. In our meta-analysis, the overall incidence of hyperdense area after thrombectomy is $48.62 \%$ (972/1999). Many factors could lead to the different rates of hyperdense area, such as different amounts of contrast agent applied, NIHSS score on admission, gender, onsetto-reperfusion time, and old generation of mechanical thrombectomy devices [18]. In fact, the definition of postinterventional hyperdense area varied in inspection time, types of CT, and other additional requirements, which could not be neglected as essential cause of different rates of hyperdense area. For instance, the inspection time changed from immediately [19] to within $24 \mathrm{~h}$ [10] in different studies after thrombectomy. The types of CT also differed from dual energy CT [16] to c-arm CT [11]. A few studies even had other requirements like CT values [10] or the location of hyperdense area [26]. Thus, we have conducted subgroup analysis according to above-confounding factors for reducing heterogeneity. As for the mechanism of hyperdense area, the most consistent point of view was that it was associated with the severity of blood-brain barrier disruption [23, 29]. If hyperdense area was due to the degradation of the basal lamina damage, the clinical manifestation was likely to be hemorrhage, and it would last for a long time; Conversely, those simply owing to some degree of endothelial damage were just 
contrast medium extravasation, and the duration of hyperdense area would be short $[25,36]$.

Several studies have described the role of hyperdense area after thrombectomy on hemorrhage, but this relationship remained controversial. Our meta-analysis suggested that hyperdense area was associated with 3.02 -fold increase in the odds of sICH. Notability, the forest graphs about the relationship between post-interventional hyperdense area and hemorrhage had great heterogeneity, which might contribute to the negative endpoint of PH2. Thus, we performed subgroup analysis according to different locations, duration, Hounsfield unit of hyperdense area and different types of CT scan as online suppl. Figures 1 and 2 displayed. Most results were unchanged, but the heterogeneity still existed. Therefore, we speculated there may be many other causes of heterogeneity, such as the quality of thrombectomy devices, the different follow-up time of hemorrhage and whether it is anterior or posterior circulation stroke. These factors were easy to be omitted and not all of them were available in the full text. For example, most studies followed up with a conventional CT or MRI $24 \mathrm{~h}$ after treatment to assess hemorrhage complications [17, 19, 20]. However, Parrilla et al. [30] followed up with nonenhanced CT in the next 48-72 h. Jang et al. [24] set the follow-up time in 3-5 days later. Obviously, the studies of Xu et al. [26] and Rouchaud et al. [17] contributed the majority of OR value with a wide range of $\mathrm{CI}$ in the outcome of HT. It may cause by the former restricting the hyperdense area to maximum CT density of $>90 \mathrm{HU}$ in the basal ganglia and the latter performing flat-panel CT immediately after completion of procedure.

Our meta-analysis suggested that patients with hyperdense area after thrombectomy were more likely to have 1.92 greater odds of poor functional outcome than the no hyperdense area group. We have also noticed that when we omitted the study of Kim et al. [23] during sensitivity analyses, the value of $i^{2}$ turn from 31 to $0 \%$. It may because all the patients Kim included underwent intra-arterial revascularization but not thrombectomy. What is more, the publication year of the research of Kim was the oldest among all 9 studies and the technological development in endovascular devices was not advanced at that time. The heterogeneity among studies may come from as follows. First, there were different criteria for measuring a poor functional outcome. Parrilla et al. [30] found no statistical difference in the final NIHSS score reduction (NIHSS pretreatment-NIHSS at discharge) and draw the conclusion that hyper-attenu-

Clinical Significance of Hyperdense Area after Endovascular Therapy ated lesions may not imply a negative clinical prognosis after thrombectomy. Therefore, we only included researches that used 90-day or discharge mRS scores as a prognostic indicator to perform this analysis. Second, patients with anterior circulation stroke were more likely to have parenchyma contrast staining on followup CT than patients with a posterior circulation infarction [16, 37]. Besides, for patients with posterior circulation cerebral infarction, the occurrence of postoperative hyperdense area was also related to poor clinical outcome [38, 39]. Accordingly, we specifically compiled patients with only anterior circulation stroke and found the presenting of hyperdense area after thrombectomy was associated with a 2.72 -fold increase in the odds of poor outcome. Third, as we all know, the higher the TICI (Thrombolysis in Cerebral Infarction) grade, the better the outcomes after thrombectomy [40]. Contrast extravasation occurred frequently in patients with failed recanalization as the contrast medium was not able to enter circulation and thereby manifested as a hyperdense lesion with disruption to the BBB [10]. For this reason, no significant association between hyperdense area and poor functional outcomes was found in the study by Lummel et al. [18].

The strengths of our meta-analysis include strict compliance with the PRISMA guidelines, prior registration with PROSPERO(CRD42020164165), and the first quantitative synthesis of available data on the effect of hyperdense area after thrombectomy for patients with AIS. Moreover, we have systematically summarized the effect of post-interventional hyperdense area on particular subtypes of HT in detail. It may be an implication for optimization of antithrombotic therapy strategy for patients with hyperdense area after endovascular treatment.

Our meta-analysis still has several limitations. First, only 16 retrospective studies were included, so the publication bias could not be avoided and the quality of included literature was not high. Second, the types of CT, follow-up time, and definition of hyperdense area differed in studies. However, we decreased this influence by performing series of subgroup analyses, and it did not modify the pooled estimated effects. Third, potential confounders, including the device and methods for endovascular treatment, the slice thickness of CT, stroke severity, stroke etiology, and onset-to-treatment time, may have been the underlying reasons for the heterogeneity among studies and reduced comparability between included studies.

In summary, there is great room for improvement in the overall quality of researches in this field. We hope that 
studies in the future can adopt a unified definition of hyperdense area to explore its clinical significance. We also anticipate that our meta-analysis can provide a reference for optimization of antithrombotic therapy strategies for patients with hyperdense area after thrombectomy.

\section{Conclusion}

Our results indicated that the presence of hyperdense area on CT after thrombectomy was associated with high risk of symptomatic intracerebral hemorrhage, poor functional outcome, as well as mortality in patients with AIS. However, further studies of high quality are needed.

\section{Statement of Ethics}

This meta-analysis protocol was approved by the Research Ethics Committee of The Second Affiliated Hospital of Soochow University.

\section{Conflict of Interest Statement}

There are no conflicts of interest.

\section{Funding Sources}

This work was supported in part by grants from the National Natural Science Foundation of China (81901198), the Basic research of Suzhou Medical and health care (SYS201724 and SYS2018061), Natural Science Foundation for Higher Education of Jiangsu Province of China (19KJB320004), and Discipline Construction Program of the Second Affiliated Hospital of Soochow University (XKTJ-XK202001 and XKTJ-TD202004). This work was also partly supported by the Young Elite Scientists Sponsorship Program by China Association for Science and Technology (2018QNRC001) and the Project of Suzhou Science and Technology Development Plan 2018 (sys2018061).

\section{Author Contributions}

Concept and design: S.J.Y. and G.D.X. Acquisition, analysis, or interpretation of data: Q.M.J., J.H., and J.G. Drafting of the manuscript: Q.M.J. and S.J.Y. Critical revision of the manuscript for important intellectual content: G.D.X., Z.C.H., H.S.W., and Y.J.C. Statistical analysis: Q.M.J. and Z.L.G.

\section{References}

1 Jadhav AP, Campbell BCV. Ongoing advances in medical and interventional treatments of large vessel occlusion stroke. Stroke. 2021 Mar;52(3):1115-1117.

2 Rocha M, Jadhav AP, Jovin TG. Endovascular therapy for large vessel occlusion stroke: an update on the most recent clinical trials. J Cereb Blood Flow Metab. 2019 Sep;39(9): 1661-3.

3 Nogueira RG, Jadhav AP, Haussen DC, Bonafe A, Budzik RF, Bhuva P, et al. Thrombectomy 6 to 24 hours after stroke with a mismatch between deficit and infarct. N Engl J Med. 2018 Jan 4;378(1):11-21.

4 Albers GW, Marks MP, Kemp S, Christensen S, Tsai JP, Ortega-Gutierrez S, et al. Thrombectomy for stroke at 6 to 16 hours with selection by perfusion imaging. N Engl J Med. 2018 Feb 22;378(8):708-18.

5 Goyal M, Menon BK, van Zwam WH, Dippel DW, Mitchell PJ, Demchuk AM, et al. Endovascular thrombectomy after large-vessel ischaemic stroke: a meta-analysis of individual patient data from five randomised trials. Lancet. 2016 Apr 23;387(10029):1723-31.

6 Tijssen MP, Hofman PA, Stadler AA, van Zwam W, de Graaf R, van Oostenbrugge RJ, et al. The role of dual energy CT in differentiating between brain haemorrhage and contrast medium after mechanical revascularisation in acute ischaemic stroke. Eur Radiol. 2014 Apr;24(4):834-40.
7 Dekeyzer S, Nikoubashman O, Lutin B, De Groote J, Vancaester E, De Blauwe S, et al. Distinction between contrast staining and hemorrhage after endovascular stroke treatment: one CT is not enough. J Neurointerv Surg. 2017 Apr;9(4):394-8.

8 Nikoubashman O, Jablawi F, Dekeyzer S, Oros-Peusquens AM, Abbas Z, Lindemeyer J, et al. MRI appearance of intracerebral iodinated contrast agents: is it possible to distinguish extravasated contrast agent from hemorrhage? AJNR Am J Neuroradiol. 2016 Aug; 37(8):1418-21.

9 Khalilzadeh O, Sabel B, Sung Y, Parikh A, Phan CM, Dinkel J, et al. Temporal evolution of intraparenchymal hyperdensity after intraarterial therapy in patients with ischemic stroke: optimal discrimination between hemorrhage and iodinated contrast. Clin Neuroradiol. 2014 Dec;24(4):365-71.

10 Chen Z, Zhang Y, Su Y, Sun Y, He Y, Chen H. Contrast extravasation is predictive of poor clinical outcomes in patients undergoing endovascular therapy for acute ischemic stroke in the anterior circulation. J Stroke Cerebrovasc Dis. 2020 Jan;29(1):104494.

11 Chen WH, Yi TY, Wu YM, Zhang MF, Lin DL, Lin XH. Parenchymal hyperdensity on $\mathrm{C}$-arm CT images after endovascular therapy for acute ischaemic stroke predicts a poor prognosis. Clin Radiol. 2019 May;74(5):399404.
12 Renú A, Amaro S, Laredo C, Román LS, Llull L, Lopez A, et al. Relevance of blood-brain barrier disruption after endovascular treatment of ischemic stroke: dual-energy computed tomographic study. Stroke. 2015 Mar; 46(3):673-9.

13 Yang M, Huo X, Gao F, Wang A, Ma N, Shi $\mathrm{H}$, et al. Low-dose rescue tirofiban in mechanical thrombectomy for acute cerebral largeartery occlusion. Eur J Neurol. 2020 Jun; 27(6):1056-61.

14 Sun C, Li X, Zhao Z, Chen X, Huang C, Li X, et al. Safety and efficacy of tirofiban combined with mechanical thrombectomy depend on ischemic stroke etiology. Front Neurol. 2019 Oct 29;10:1100.

15 Zhao W, Che R, Shang S, Wu C, Li C, Wu L, et al. Low-Dose tirofiban improves functional outcome in acute ischemic stroke patients treated with endovascular thrombectomy. Stroke. 2017 Dec;48(12):3289-94.

16 An H, Zhao W, Wang J, Wright JC, Elmadhoun $\mathrm{O}, \mathrm{Wu} \mathrm{D}$, et al. Contrast staining may be associated with intracerebral hemorrhage but not functional outcome in acute ischemic stroke patients treated with endovascular thrombectomy. Aging Dis. 2019 Aug 1;10(4): 784-92. 
17 Rouchaud A, Pistocchi S, Blanc R, Engrand N, Bartolini B, Piotin M. Predictive value of flatpanel CT for haemorrhagic transformations in patients with acute stroke treated with thrombectomy. J Neurointerv Surg. 2014 Mar;6(2):139-43.

18 Lummel N, Schulte-Altedorneburg G, Bernau C, Pfefferkorn T, Patzig M, Janssen $\mathrm{H}$, et al. Hyperattenuated intracerebral lesions after mechanical recanalization in acute stroke. AJNR Am J Neuroradiol. 2014 Feb;35(2): 345-51.

19 Desilles JP, Rouchaud A, Labreuche J, Meseguer E, Laissy JP, Serfaty JM, et al. Bloodbrain barrier disruption is associated with increased mortality after endovascular therapy. Neurology. 2013 Feb 26;80(9):844-51.

20 Yoon W, Seo JJ, Kim JK, Cho KH, Park JG, Kang HK. Contrast enhancement and contrast extravasation on computed tomography after intra-arterial thrombolysis in patients with acute ischemic stroke. Stroke. 2004 Apr; 35(4):876-81.

21 Payabvash S, Qureshi MH, Taleb S, Pawar S, Qureshi AI. Middle cerebral artery residual contrast stagnation on noncontrast CT scan following endovascular treatment in acute ischemic stroke patients. J Neuroimaging. 2015 Nov-Dec;25(6):946-51.

22 Payabvash S, Qureshi MH, Khan SM, Khan M, Majidi S, Pawar S, et al. Differentiating intraparenchymal hemorrhage from contrast extravasation on post-procedural noncontrast CT scan in acute ischemic stroke patients undergoing endovascular treatment. Neuroradiology. 2014 Sep;56(9):737-44.

23 Kim JT, Heo SH, Cho BH, Choi SM, Lee SH, Park MS, et al. Hyperdensity on non-contrast CT immediately after intra-arterial revascularization. J Neurol. 2012 May;259(5):936-43.

24 Jang YM, Lee DH, Kim HS, Ryu CW, Lee JH, Choi CG, et al. The fate of high-density lesions on the non-contrast CT obtained immediately after intra-arterial thrombolysis in ischemic stroke patients. Korean J Radiol. 2006 Oct-Dec;7(4):221-8.
25 Nakano S, Iseda T, Kawano H, Yoneyama T, Ikeda T, Wakisaka S. Parenchymal hyperdensity on computed tomography after intra-arterial reperfusion therapy for acute middle cerebral artery occlusion: incidence and clinical significance. Stroke. 2001 Sep;32(9):2042-8.

26 Xu C, Zhou Y, Zhang R, Chen Z, Zhong W, Gong X, et al. Metallic hyperdensity sign on noncontrast CT immediately after mechanical thrombectomy predicts parenchymal hemorrhage in patients with acute large-artery occlusion. AJNR Am J Neuroradiol. 2019 Apr;40(4):661-7.

27 Shi ZS, Duckwiler GR, Jahan R, Tateshima S, Szeder V, Saver JL, et al. Early blood-brain barrier disruption after mechanical thrombectomy in acute ischemic stroke. J Neuroimaging. 2018 May;28(3):283-8.

28 Kim JM, Park KY, Lee WJ, Byun JS, Kim JK, Park MS, et al. The cortical contrast accumulation from brain computed tomography after endovascular treatment predicts symptomatic hemorrhage. Eur J Neurol. 2015 Nov; 22(11):1453-8.

29 Nikoubashman O, Reich A, Gindullis M, Frohnhofen K, Pjontek R, Brockmann MA, et al. Clinical significance of post-interventional cerebral hyperdensities after endovascular mechanical thrombectomy in acute ischaemic stroke. Neuroradiology. 2014 Jan; 56(1):41-50.

30 Parrilla G, García-Villalba B, Espinosa de Rueda M, Zamarro J, Carrión E, HernándezFernández $\mathrm{F}$, et al. Hemorrhage/contrast staining areas after mechanical intra-arterial thrombectomy in acute ischemic stroke: imaging findings and clinical significance. AJNR Am J Neuroradiol. 2012 Oct;33(9):1791-6.

31 Wildenhain SL, Jungreis CA, Barr J, Mathis J, Wechsler L, Horton JA. CT after intracranial intraarterial thrombolysis for acute stroke. AJNR Am J Neuroradiol. 1994 Mar;15(3): 487-92.

32 Larrue V, von Kummer R, del Zoppo G, Bluhmki E. Hemorrhagic transformation in acute ischemic stroke. Potential contributing factors in the European Cooperative Acute Stroke Study. Stroke. 1997 May;28(5):957-60.
33 Stang A. Critical evaluation of the NewcastleOttawa scale for the assessment of the quality of nonrandomized studies in meta-analyses. Eur J Epidemiol. 2010 Sep;25(9):603-5.

34 Kim H, Lee SJ, Lee TK, Jung KO. Subarachnoid contrast accumulation and Alberta Stroke Program Early Computed Tomography Score applied to contrast accumulation after thrombectomy as predictors of symptomatic hemorrhage. World Neurosurg. 2020 Jun;138:e847-58.

35 Komiyama M, Nishijima Y, Nishio A, Khosla VK. Extravasation of contrast medium from the lenticulostriate artery following local intracarotid fibrinolysis. Surg Neurol. 1993 Apr;39(4):315-9.

36 Nakano S, Iseda T, Yoneyama T, Wakisaka S. Early CT signs in patients with acute middle cerebral artery occlusion: incidence of contrast staining and haemorrhagic transformations after intra-arterial reperfusion therapy. Clin Radiol. 2006 Feb;61(2):156-62.

37 Morhard D, Ertl L, Gerdsmeier-Petz W, ErtlWagner B, Schulte-Altedorneburg G. Dualenergy CT immediately after endovascular stroke intervention: prognostic implications. Cardiovasc Intervent Radiol. 2014 Oct;37(5): 1171-8.

38 Sun Y, Su Y, Chen Z, He Y, Zhang Y, Chen H. Contrast extravasation after endovascular treatment in posterior circulation stroke. World Neurosurg. 2019 Oct;130:e583-7.

39 Son S, Kim YW, Oh MK, Kim SK, Park KJ, Choi NC, et al. Initial factors affecting the clinical outcome after successful recanalization via MR-based mechanical thrombectomy in patients with acute ischemic stroke due to basilar artery occlusion. I Neurointerv Surg. 2016 Sep;8(9):889-93.

40 Jang KM, Nam TK, Ko MJ, Choi HH, Kwon JT, Park SW, et al. Thrombolysis in cerebral infarction grade $2 \mathrm{C}$ or 3 represents a better outcome than 2B for endovascular thrombectomy in acute ischemic stroke: a network meta-analysis. World Neurosurg. 2020 Apr;136: e419-39. 